\title{
Microsurgical anterior cervical foraminotomy for radiculopathy: a new approach to cervical disc herniation
}

\author{
Hae-Dong Jho, M.D., Ph.D. \\ Department of Neurological Surgery, University of Pittsburgh School of Medicine, Pittsburgh, \\ Pennsylvania
}

A new technique of microsurgical anterior foraminotomy was developed to improve the treatment of cervical radiculopathy. This technique provides direct anatomical decompression of the compressed nerve root by removing the compressive spondylotic spur or disc fragment. The nerve root is decompressed from its origin in the spinal cord to the point at which it passes behind the vertebral artery laterally. Because most of the disc within the intervertebral space is undisturbed, a functioning motion segment of the disc remains intact. This technique differs from that of Verbiest in that it does not directly transpose the vertebral artery. Unlike Hakuba's technique, the disc within the intervertebral disc space is not removed.

Key Words * cervical vertebra * discectomy * intervertebral disc displacement * radiculopathy * spine * spinal nerve roots

Cervical disc disorders causing radiculopathy have been treated surgically from either an anterior or a posterior approach. In 1934, Mixter and Barr[9] reported on 19 ruptured intervertebral discs treated successfully with a posterior laminectomy. Four of these patients had cervical disc herniation. Since then, the posterior approach for discogenic cervical radiculopathy has evolved into a small keyhole foraminotomy with or without discectomy.[5,11] Although the posterior procedure preserves a functioning motion segment, decompression of the nerve root is indirect, leaving the compressive lesion untouched. In addition, to avoid excess epidural bleeding, the preferable posterior approach is performed with the patient sitting, which has known risks.[2]

The anterior approach was developed in the 1950s to achieve direct decompression of the nerve root by removing the compressing spondylotic spur and the disc fragment located anterior to the nerve root. $[1,10]$ Anterior cervical discectomy with or without bone fusion has been widely adopted for the treatment of cervical radiculopathy. The reported surgical outcome has been comparable to that achieved using the posterior approach.[4,5,7,11] However, even if bone fusion is not performed intraoperatively, it will eventually occur after an anterior cervical discectomy, thereby eliminating the functioning motion segment.[3,4,8] Bone fusion with elimination of a motion segment is not a physiological treatment.

This report describes a new microsurgical technique developed to accomplish direct nerve root 
decompression via the anterior approach while preserving the functioning motion segment as the posterior approach does. Being an anterolateral approach, the technique is similar to that of Verbiest[13] or Hakuba[3] yet still quite different from those procedures with regard to intervertebral disc preservation and simplicity of technique.

\section{CLINICAL MATERIAL AND METHODS}

Before this surgical procedure was used in patients, it was practiced on cadavers. The goal was to achieve direct effective anatomical decompression of the nerve root and preserve the functioning motion segment.

\section{Operative Technique}

The operation is performed with the patient under general anesthesia induced by means of endotracheal intubation. The position of the patient is similar to that used for an anterior cervical discectomy. With the patient supine, a bolster is placed behind both shoulders to maintain gentle extension of the cervical spine. The head is positioned with the midline upright. Both shoulders are gently pulled caudally and fixed with tape to facilitate a good lateral view of the cervical spine on the intraoperative roentgenogram. A cervical traction device is not used. The entire anterior neck is prepared with antiseptic solution and draped.

A transverse incision, 3 to $5 \mathrm{~cm}$ long, is made in a skin crease ipsilateral to the radiculopathy; the incision is similar to that used for an anterior cervical discectomy. The first two-thirds of the incision is medial to the sternocleidomastoid muscle and the remainder is lateral to the medial border of that muscle. The subcutaneous tissue and the platysma muscle are incised along the line of the skin incision. The loose connective tissue layer under the platysma muscle is cleanly undermined to provide space in which to work. A combination of sharp and blunt dissection is used to access the anterior column of the cervical spine, keeping the carotid artery and the sternocleidomastoid muscle lateral and the strap muscle, trachea, and esophagus medial. The prevertebral fascia is opened and the anterior column of the cervical spine is exposed. The correct level is then confirmed radiographically. Thus far, the procedure is similar to that used for an anterior cervical discectomy.

Next, an anterior cervical discectomy retractor system is applied. Only smooth-tipped retractor blades are used, thus avoiding injury to the trachea and esophagus medially and the carotid artery and vagus nerve laterally. Retraction will naturally expose the ipsilateral longus colli muscle rather than the midline anterior disc surface (Fig. 1A). Use of the operating microscope begins at this stage. The medial portion of the longus colli muscle is excised to expose the medial parts of the transverse processes of the upper and lower vertebrae (Fig. 1B). The vertebral artery is exposed anterior to the C-7 transverse process after the longus colli is excised. Therefore, when operating at C6-7, care must be taken not to injure the vertebral artery while removing the medial portion of that muscle. For operations above C6-7, the vertebral artery is purposefully not exposed at this point. 


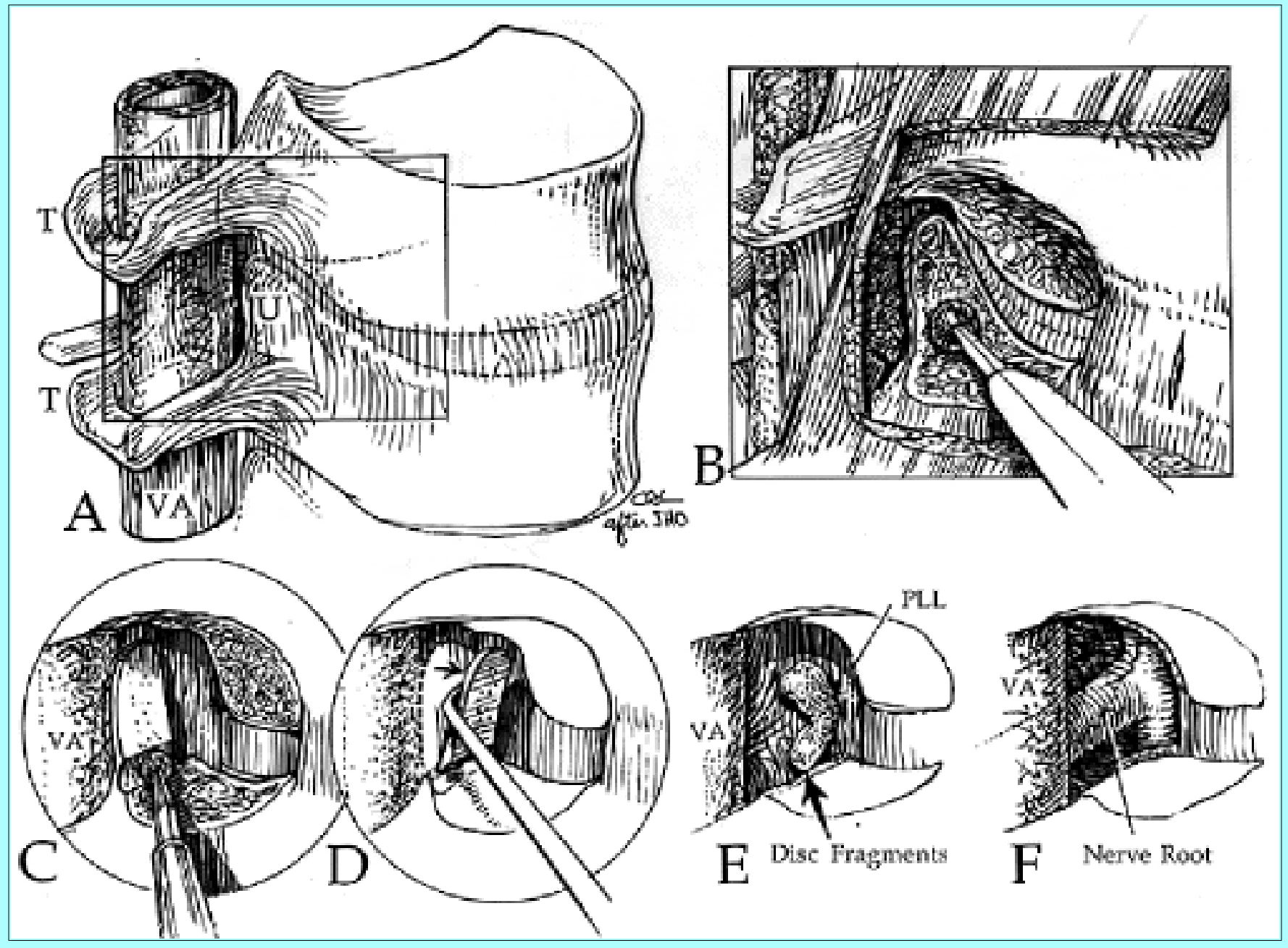

Fig. 1. Schematic drawings displaying microsurgical anterior foraminotomy. PLL = posterior longitudinal ligament; $\mathrm{T}=$ transverse process; $\mathrm{VA}=$ vertebral artery; and $\mathrm{U}=$ uncinate process. A: An overview of cervical structures seen in the anterior approach. The surgical exposure is approximated in the box and enlarged in subsequent illustrations. B: The medial portion of the longus colli muscle is excised to expose the uncovertebral joint and the medial portion of the upper and lower transverse processes. C: Under an operating microscope, the uncovertebral joint is drilled up to the posterior longitudinal ligament. D: A piece of the thin cortical bone (small arrow) of the uncinate process covering the vertebral artery is fractured and removed. The vertebral artery can be identified by its pulsation. E: The compressed nerve root is distended forward by bone decompression. A tail of the herniated disc may be visible through a tear in the posterior longitudinal ligament (large arrow). F: The posterior longitudinal ligament is removed to confirm decompression of the nerve root. The nerve root is then visible from its origin in the spinal cord to its exit behind the vertebral artery. Removal of the posterior longitudinal ligament may not be necessary if it has no defect.

Once the medial parts of the transverse processes of the upper and lower vertebrae have been identified, the ipsilateral uncovertebral joint between them can be seen. The interface of the uncovertebral joint will be angled approximately 30 š cephalad from the horizontal line of the intervertebral disc. Next, a sharp vertical incision of the disc is made at the medial margin of the ipsilateral uncovertebral joint to prevent inadvertent internal disruption of the remaining disc. While drilling the bone, repeated sharp cutting of the deeper disc may be required. The thin layer of disc material located at the uncovertebral joint is 
removed.

The uncovertebral joint is drilled between the transverse processes using a high-speed microdrill attached to an angled handpiece (Fig. 1B). To prevent injury to the vertebral artery, the thin cortical bone is left attached to the ligamentous tissue covering the medial portion of this artery; drilling continues down to the posterior longitudinal ligament (Fig. 1C). As drilling advances posteriorly, the direction of the drilling is gently inclined medially. When the posterior longitudinal ligament is exposed, a piece of the thin cortical bone remains attached lateral to the ligamentous tissue covering the vertebral artery. This lateral remnant of the uncinate process is dissected from the ligamentous tissue and fractured at the base of uncinate process (Fig. 1D). Then it is further dissected from the surrounding soft tissue and removed, enabling identification of the vertebral artery by its pulsation between the transverse processes of the vertebrae. Drilling at the base of the uncinate process must proceed cautiously because the nerve root lies just behind it. After the uncinate process becomes loosened at its base, it is safer to remove the remaining thin bone of the uncinate process by fracturing it from its base than by continued drilling. When the remaining piece of the uncinate process is removed, the compressed nerve root will be distended forward by bone decompression (Fig. 1E). The size of the hole that is made by drilling at the uncovertebral joint is usually approximately 5 to $8 \mathrm{~mm}$ wide transversely by 7 to $10 \mathrm{~mm}$ vertically. If there is no ruptured herniated disc fragment behind the posterior longitudinal ligament, this will be the end of the nerve root decompression.

At this point the posterior longitudinal ligament still covers the nerve root and the lateral margin of the spinal cord. If the disc fragment has ruptured through the posterior longitudinal ligament, the tail of the disc material will be visible at this stage and this disc fragment can be removed. To avoid overlooking a hidden disc fragment, the ligament is incised with a No. 15 blade knife or microscissors and removed with a 1- or 2-mm foot-plated bone punch. The ipsilateral one-third to one-half of the spinal cord is thus exposed and the nerve root can be seen laterally as it exits behind the vertebral artery (Fig. 1F).

Excision of the posterior longitudinal ligament has been performed selectively in more recent cases because it is unlikely that there is a hidden ruptured disc fragment without disruption of this ligament. Removal of the ligament sometimes is complicated by cumbersome epidural bleeding. If the ligament is not removed, the procedure is much simpler and operating time is shorter. When epidural bleeding does occur during removal of the posterior longitudinal ligament, it usually can be stopped with bipolar coagulation. To avoid any potential compressive material coming in contact with the nerve root or spinal cord, hemostatic agents are not used. Although epidural bleeding is usually not a problem, removing the posterior longitudinal ligament is the most difficult part of this procedure. The disc within the intervertebral space remains untouched and preserved.

Finally, the platysma is closed with interrupted 3-0 absorbable stitches and the skin is approximated with subcuticular sutures. The operation can be performed at multiple levels, if required. To minimize postoperative incisional pain, a few milliliters of local anesthetic is injected subcutaneously. A cervical collar is not used. In earlier cases, the patient was kept in the hospital overnight, but the operation is now performed as an outpatient procedure.

\section{RESULTS}

\section{Case Illustration}

This 44-year-old woman was referred to the University of Pittsburgh Medical Center in July 1993 
complaining of symptoms that had persisted since a motor vehicle accident in October 1989. She suffered from constant severe neck pain, interscapular pain, and radicular pain along the left upper extremity extending to the thumb and index finger of the left hand. Her symptoms were not relieved by analgesic medications, physical therapy, or chiropractic manipulations. On examination, she had mild weakness (4/5) of the left deltoid and biceps muscles, a decreased left biceps reflex, and hypalgesia along the C-6 dermatome in the left forearm and hand. Magnetic resonance (MR) imaging of the cervical spine showed C5-6 disc herniation encroaching on the nerve root at the left C5-6 neural foramen (Fig. 2).

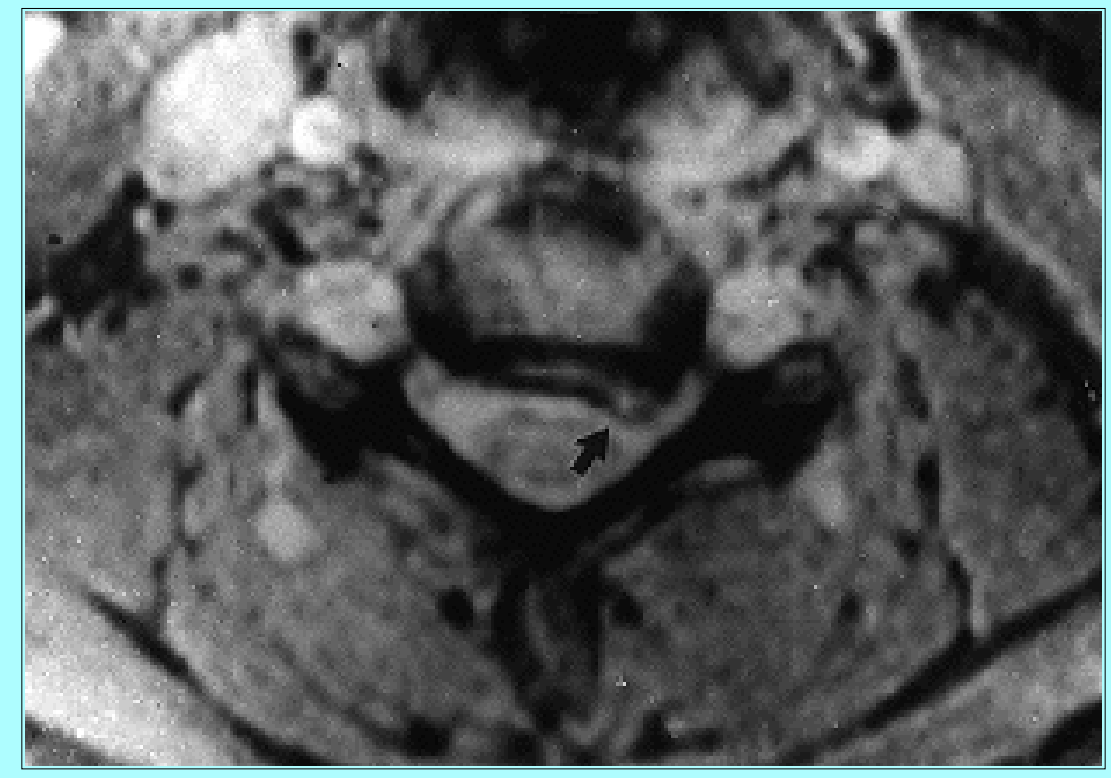

Fig. 2. Magnetic resonance image of the cervical spine showing the disc and spondylotic spur encroaching on the left C-6 nerve root at the C5-6 neural foramen (arrow).

In July 1993, the patient underwent a C5-6 microsurgical anterior foraminotomy via a left-sided approach. The operation was performed on an outpatient basis. The patient was free of symptoms with a normal range of motion in the neck and minimal discomfort from the incision a few hours after the operation. A cervical collar was not used. At a 4-week follow-up visit the patient was fully recovered. Specifically, her neck, interscapular, and left C-6 radicular pain had disappeared; the motor strength in her biceps and deltoid muscles had improved; and a normal range of motion in her neck was maintained. Postoperative MR imaging of the cervical spine showed a good decompression of the left C-6 neural foramen in the axial view and preservation of the remaining disc at C5-6 in the sagittal view (Fig. 3). 


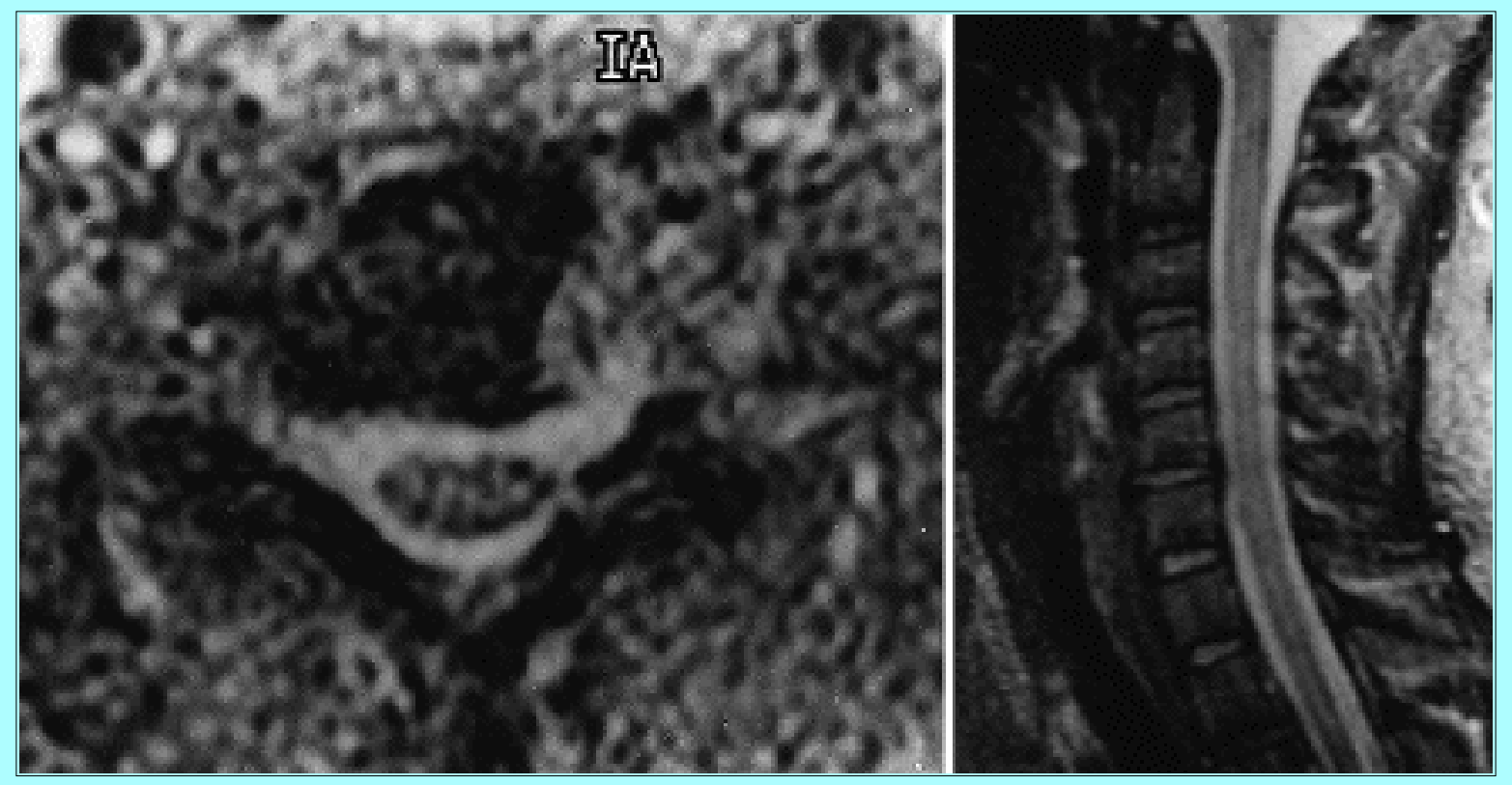

Fig. 3. Postoperative magnetic resonance images of the cervical spine demonstrating a good decompression of the left C-6 nerve root in the axial view (left) and maintenance of the intervertebral disc at C5-6 in the sagittal view (right).

Postoperative anteroposterior and oblique roentgenograms of the cervical spine showed enlargement of the neural foramen by a bone resection at the left C5-6 uncovertebral joint (Fig. 4). The patient's 1-year follow-up examination showed that she was continuing to do well.

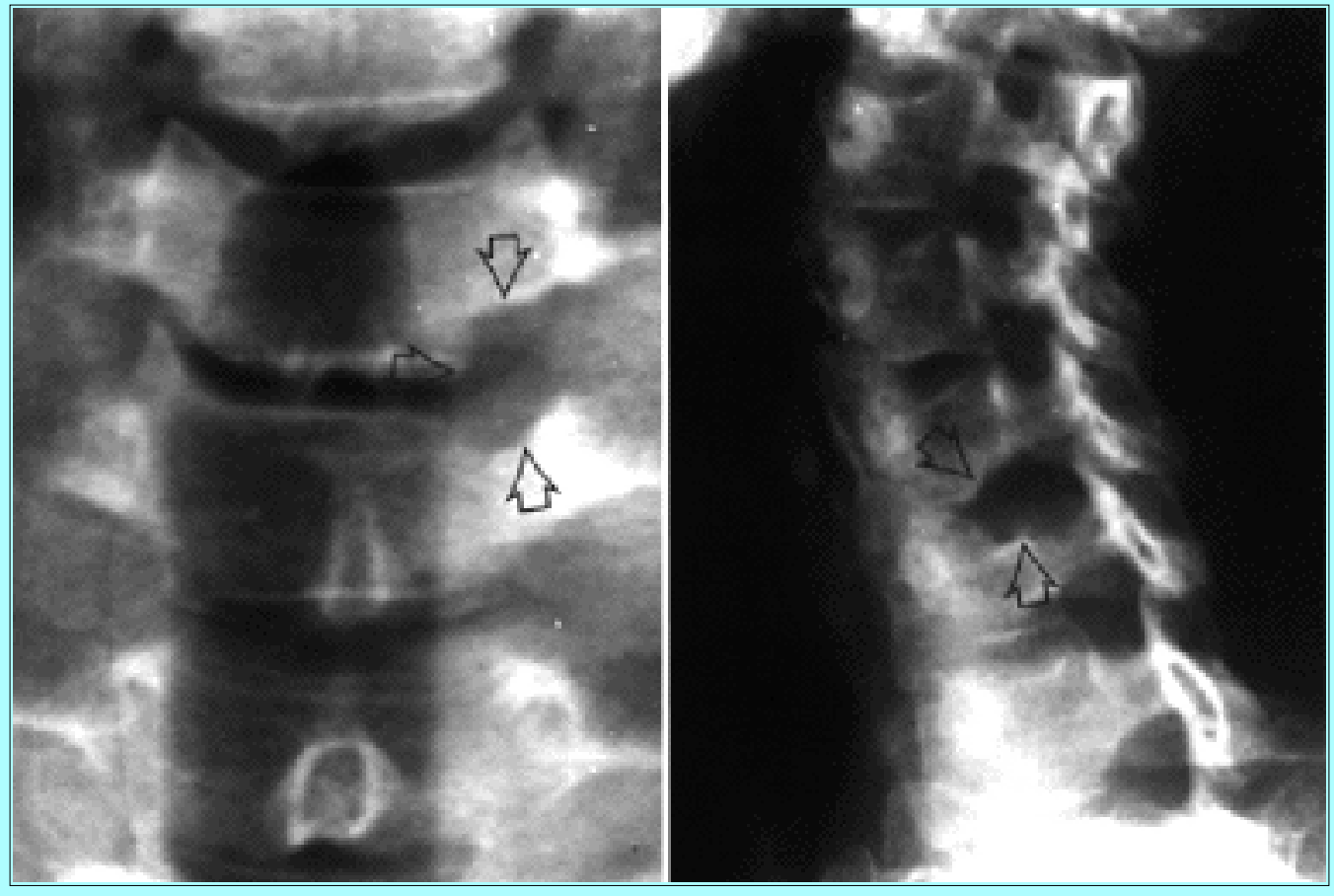

Fig. 4. Cervical spine roentgenograms, anteroposterior (left) and oblique (right) views, showing the degree of bone resection produced by microsurgical anterior foraminotomy (open arrows).

\section{DISCUSSION}

Since 1934, when Mixter and Barr[9] reported on the use of a posterior laminectomy to treat 19 cases of intervertebral disc herniation, including four ruptured cervical discs, the posterior laminectomy technique 
has evolved to a small keyhole foraminotomy.[11] Despite the good surgical outcome reported in the literature, the posterior technique is an indirect decompression that leaves the compressive lesion untouched.[5] To minimize epidural bleeding, the posterior approach is often performed with the patient sitting. The potential risks of the sitting position are known;[2] on the other hand, the posterior keyhole foraminotomy has the advantage of preserving a functioning motion segment.

To accomplish direct decompression of the compressive spondylotic spur and disc fragment, Robinson and Smith[10] in 1955 and Cloward[1] in 1958 pioneered the anterior cervical discectomy with bone fusion. Outcomes achieved using this technique reportedly have been comparable to those using the posterior approach. $[5,7,11]$ Microsurgical anterior cervical discectomy without bone fusion has achieved results similar to those for anterior cervical discectomy with interbody bone fusion.[4,7,8] Even without operative fusion, the anterior cervical discectomy leads to bone fusion, thereby eliminating the functioning unit of the intervertebral disc.[3,8] Elimination of the motion segment by bone fusion is not a physiological result.

In 1968, Verbiest[13] reported using the anterolateral approach in the treatment of cervical disc disease. His technique had been described previously for its use in exposing the vertebral segment of the vertebral artery. Through an anterolateral skin incision similar to that used in an anterior cervical discectomy, the longus colli muscle was sectioned to expose the transverse processes; these were then were removed and the vertebral artery was exposed. Following this, the vertebral artery was mobilized and displaced laterally with tape to allow access to the neural foramen and the lateral surface of the disc. The operation was performed with or without bone fusion. Despite the direct, effective anatomical decompression of the spondylotic bone spur, this surgical technique was not widely accepted, probably due to the risk of damaging the vertebral artery and the complexity of the operative technique.

The surgical technique reported here differs from that of Verbiest[13] in several ways. The longus colli muscle is sectioned only at its most medial portion to expose the beginning of the transverse processes. The transverse processes are not removed, and the vertebral artery is not mobilized. Rather than exposing the lateral surface of the disc or osteophytes by displacing the vertebral artery, spondylotic osteophytes are approached directly by microdrilling the uncinate process. Using the modern operating microscope and delicate microdrilling tools, this procedure can be performed relatively quickly and easily. Moreover, it preserves the disc in the intervertebral space, whereas Verbiest's procedure does not.

In 1976, Hakuba[3] introduced the transuncodiscal approach, which is a combined anterior and lateral approach to cervical discs. Although the lateral approach he described is similar to the technique reported here, that author sectioned the medial half of the sternocleidomastoid muscle. The longus colli muscle was sectioned and retracted craniocaudally to expose the entire anterior view of the transverse processes. In addition to resecting the uncovertebral joint, the entire disc was removed including the ipsilateral posterior osteophyte and the contralateral uncinate process. Among a series of 40 patients, 20 did not undergo interbody fusion. Nonetheless, follow-up radiographs showed solid bone fusion in most of these patients who did not undergo fusion. The procedure reported here differs from that of Hakuba in that it is designed to preserve the disc as much as possible in the hope that the remaining disc can still function as a mobile unit along with the facet joints.

In 1987, Lesoin, et al.,[6] reported on a surgical procedure similar to that of Hakuba.[3] These authors performed an anterolateral foraminotomy in addition to the formal anterior cervical discectomy in four patients with lateral foraminal disc herniation. Bone graft fusion was performed after the discectomy. 
Snyder and Bernhardt,[12] in 1989, described an anterior cervical fractional interspace decompression for the treatment of cervical radiculopathy. Decompression was performed by drilling a 5- to 6-mm-wide cylindrical hole with a high-speed 5-mm burr in the lateral one-third of the intervertebral disc. The drilling proceeded in an anteroposterior and slightly mediolateral trajectory to intersect the uncinate process at the level of the neural foramen. The final decompression of the nerve root was accomplished with small curettes.

These authors' surgical strategy was similar to mine in trying to preserve the intervertebral disc as much as possible. However, they entered the lateral one-third of the disc directly, making a small hole and leaving the lateral portion of the uncinate process untouched. The decompression was limited to a 3-mm radius around the nerve root. Thus, rather than an "anterior cervical fractional interspace decompression," their procedure more accurately might be described as a "lateral one-third limited anterior cervical discectomy." Our approach is completely lateral, exposing the vertebral artery when the entire uncinate process is removed. It exposes the lateral portion of the spinal cord medially and the nerve root exiting behind the vertebral artery laterally to allow decompression of this entire segment of the nerve root.

The surgical technique described here emphasizes preservation of the intervertebral disc as a functioning motion segment. Despite the effort to preserve the motion segment, however, bone fusion at the involved level eventually may result from a combination of the preexisting discogenic degenerative process and surgical trauma. The ultimate outcome of the motion segment at the involved level remains to be seen in long-term follow-up studies.

Although injury to the vertebral artery did not occur in a series of more than 30 cases, it would seem to be the main potential risk of this technique. The first potential site of vertebral artery injury is at the C6-7 level, where the vertebral artery runs between the transverse process of $\mathrm{C}-7$ and the longus colli muscle. To avoid this injury, the longus colli muscle should be incised at the level of the C-6 transverse process. When the muscle stump is reflected toward the C-7 transverse process caudally, the surgeon will see the vertebral artery beneath the longus colli muscle. The artery then enters the transverse foramen of C- 6 . The second potential site of injury to the vertebral artery is where it passes lateral to the uncinate process. Injury here can be avoided by leaving the thin cortical bone of the uncinate process when the uncovertebral joint is drilled. The remaining thin cortical bone should be dissected from the ligamentous tissue that still covers the vertebral artery. The third potential site of injury is at the transverse foramen. Care should be taken not to enter the transverse foramen while the uncovertebral joint is drilled.

Despite the potential risk of injury to the vertebral artery outlined above, which rarely occurs with a conventional anterior cervical discectomy, the ability to view the artery directly with this new technique may actually reduce the risk of injury. The frequency of vertebral artery injury with this approach remains to be defined.

This new technique accomplishes a direct resection of the compressive lesion, that is, the soft disc fragment, the spondylotic spur, or both. Although long-term follow-up data are not yet available, the short-term outcome has been encouraging for the more than 30 patients with a cervical radiculopathy treated using this technique. Most patients had good resolution of radicular, posterior neck, and interscapular pain and their postoperative range of motion in the neck was unrestricted. Usually, the operation was performed on an outpatient basis. A biomechanical laboratory study will be required to measure the actual changes in cervical spine stability. This report simply describes the technique of microsurgical anterior foraminotomy, which provides direct decompression of the nerve root and 
preservation of the intervertebral disc. The short-term follow-up data will be reported elsewhere.

\section{Acknowledgments}

The author wishes to thank Helene W. Marion for editorial assistance and Sandra Lash for assistance with preparation of the manuscript.

\section{References}

1. Cloward RB: The anterior approach for removal of ruptured cervical disks. J Neurosurg 15:602-614, 1958

2. Ehni B, Ehni G, Patterson RH Jr: Epidural spinal cord and nerve root compression from benign lesions of the cervical area, in Youmans JR (ed): Neurological Surgery, ed 3. Philadelphia: WB Saunders, 1990, Vol 4, pp 2905-2906

3. Hakuba A: Trans-unco-discal approach. A combined anterior and lateral approach to cervical discs. J Neurosurg 45:284-291, 1976

4. Hankinson HL, Wilson CB: Use of the operating microscope in anterior cervical discectomy without fusion. J Neurosurg 43:452-456, 1973

5. Henderson CM, Hennessy RG, Shuey HM Jr, et al: Posterior-lateral foraminotomy as an exclusive operative technique for cervical radiculopathy: a review of 846 consecutively operated cases.

Neurosurgery 13:504-512, 1983

6. Lesoin F, Biondi A, Jomin M: Foraminal cervical herniated disc treated by anterior discoforaminotomy. Neurosurgery 21:334-338, 1987

7. Lunsford LD, Bissonette DJ, Jannetta PJ, et al: Anterior surgery for cervical disc disease. Part 1:

Treatment of lateral cervical disc herniation in 253 cases. J Neurosurg 53:1-11, 1980

8. Martins AN: Anterior cervical discectomy with and without interbody bone graft. J Neurosurg 44:290-295, 1976

9. Mixter WJ, Barr JS: Rupture of the intervertebral disc with involvement of the spinal canal. N Engl J Med 211:210-215, 1934

10. Robinson RA, Smith GW: Anterolateral cervical disc removal and interbody fusion for cervical disc syndrome. Bull Johns Hopkins Hosp 96:223-224, 1955 (Abstract)

11. Scoville WB, Dohrmann GJ, Corkill G: Late results of cervical disc surgery. J Neurosurg 45:203-210, 1976

12. Snyder GM, Bernhardt AM: Anterior cervical fractional interspace decompression for treatment of cervical radiculopathy. A review of the first 66 cases. Clin Orthop 246:92-99, 1989

13. Verbiest H: A lateral approach to the cervical spine: technique and indications. J Neurosurg 28:191-203, 1968

Manuscript received January 9, 1995. 
Accepted in final form June 22, 1995.

Address reprint requests to: Hae-Dong Jho, M.D., Ph.D., Department of Neurological Surgery, Presbyterian University Hospital, Suite B-400, 200 Lothrop Street, Pittsburgh, Pennsylvania 15213. This article appeared in the February 1996 issue of Journal of Neurosurgery. 